\title{
Proof of the combinatorial nullstellensatz over integral domains, in the spirit of Kouba
}

\author{
Peter Heinig* \\ Lehr- und Forschungseinheit M9 \\ für Angewandte Geometrie und Diskrete Mathematik, \\ Zentrum Mathematik, Technische Universität München, \\ Boltzmannstraße 3, D-85748 Garching bei München, Germany \\ heinig@ma.tum.de \\ Submitted: Jan 4, 2010; Accepted: Feb 11, 2010; Published: Feb 22, 2010 \\ Mathematics Subject Classification 2010: 13G05, 15A06
}

\begin{abstract}
It is shown that by eliminating duality theory of vector spaces from a recent proof of Kouba [A duality based proof of the Combinatorial Nullstellensatz, Electron. J. Combin. 16 (2009), \#N9] one obtains a direct proof of the nonvanishing-version of Alon's Combinatorial Nullstellensatz for polynomials over an arbitrary integral domain. The proof relies on Cramer's rule and Vandermonde's determinant to explicitly describe a map used by Kouba in terms of cofactors of a certain matrix.

That the Combinatorial Nullstellensatz is true over integral domains is a wellknown fact which is already contained in Alon's work and emphasized in recent articles of Michałek and Schauz; the sole purpose of the present note is to point out that not only is it not necessary to invoke duality of vector spaces, but by not doing so one easily obtains a more general result.
\end{abstract}

\section{Introduction}

The Combinatorial Nullstellensatz is a very useful theorem (see [1]) about multivariate polynomials over an integral domain which bears some resemblance to the classical Nullstellensatz of Hilbert.

Theorem 1 (Alon, Combinatorial Nullstellensatz, ideal-containment-version, Theorem 1.1 in [1]). Let $K$ be a field, $R \subseteq K$ a subring, $f \in R\left[x_{1}, \ldots, x_{n}\right], S_{1}, \ldots, S_{n}$ arbitrary nonempty subsets of $K$, and $g_{i}:=\prod_{s \in S_{i}}\left(x_{i}-s\right)$ for every $1 \leqslant i \leqslant n$. If $f\left(s_{1}, \ldots, s_{n}\right)=0$

\footnotetext{
*The author was supported by a scholarship from the Max Weber-Programm Bayern and by the ENB graduate program TopMath.
} 
for every $\left(s_{1}, \ldots, s_{n}\right) \in S_{1} \times \cdots \times S_{n}$, then there exist polynomials $h_{i} \in R\left[x_{1}, \ldots, x_{n}\right]$ with the property that $\operatorname{deg}\left(h_{i}\right) \leqslant \operatorname{deg}(f)-\operatorname{deg}\left(g_{i}\right)$ for every $1 \leqslant i \leqslant n$, and $f=\sum_{i=1}^{n} h_{i} g_{i}$.

Theorem 2 (Alon, Combinatorial Nullstellensatz, nonvanishing-version, Theorem 1.2 in [1]). Let $K$ be a field, $R \subseteq K$ a subring, and $f \in R\left[x_{1}, \ldots, x_{n}\right]$. Let $c \cdot x_{1}^{d_{1}} \cdots x_{n}^{d_{n}}$ be a term in $f$ with $c \neq 0$ whose degree $d_{1}+\cdots+d_{n}$ is maximum among all degrees of terms in $f$. Then every product $S_{1} \times \cdots \times S_{n}$, where each $S_{i}$ is an arbitrary finite subset of $R$ satisfying $\left|S_{i}\right|=d_{i}+1$, contains at least one point $\left(s_{1}, \ldots, s_{n}\right)$ with $f\left(s_{1}, \ldots, s_{n}\right) \neq 0$.

Three comments are in order. First, talking about subrings of a field is equivalent to talking about integral domains: every subring of a field clearly is an integral domain, and, conversely, every integral domain $R$ is (isomorphic to) a subring of its field of fractions Quot $(R)$. Second, strictly speaking, rings are mentioned in [1] only in Theorem 1, but Alon's proof in [1] of Theorem 2 is valid for polynomials over integral domains as well. Third, it is intended that the $S_{i}$ are allowed to be subsets of $K$ in Theorem 1 but required to be subsets of $R$ in Theorem 2, but this is done only for convenience. Theorem 2 is easily seen to be equivalent to the formulation obtained when 'arbitrary finite subset of $R$ ' is replaced by 'arbitrary finite subset of $K$ '.

In [1], Theorem 2 was deduced from Theorem 1. In [3], Kouba gave a beautifully simple and direct proof of the nonvanishing-version of the Combinatorial Nullstellensatz, bypassing the use of the ideal-containment-version. Kouba's argument was restricted to the case of polynomials over a field and at one step applied a suitably chosen linear form on the vector space $K\left[x_{1}, \ldots, x_{n}\right]$ to the given polynomial $f$ in Theorem 2 .

However, for Kouba's idea to work, it is not necessary to have recourse to duality theory of vector spaces and in the following section it will be shown how to make Kouba's idea work without it, thus obtaining a direct proof of the full Theorem 2.

Two relevant recent articles ought to be mentioned. A very short direct proof of Theorem 2 was given by Michałek in [5] who explicitly remarks that the proof works for integral domains as well. Moreover, the differences $\left\{ \pm\left(s-s^{\prime}\right):\left\{s, s^{\prime}\right\} \in\left(\begin{array}{c}S_{k} \\ 2\end{array}\right)\right\}$ in the proof below play a similar role in Michałek's proof. In [6], Schauz obtained far-reaching generalizations and sharpenings of Theorem 2, expressly working with integral domains and generalizations thereof throughout the paper.

The present author wishes to emphasize that the proof in the present paper differs from Kouba's proof only in the setting and the way in which the coefficients for Kouba's linear form are obtained and he offers the following as its raison d'être: Mathematical proofs should be treated as mathematical objects in their own right and Kouba's argument is a mathematical proof which is worth being placed into what the present author feels is its proper generality. In [3], the argument was presented under the heading of vector space duality and with explicit reference to (dual) bases - notions which essentially depend on the ability to uniquely invert the scalar multiplication operation when the scalar ring of a module is a field. Casual readers hence might get away with the impression that Kouba's argument essentially rests on those things. However, the argument emerges unscathed and in greater generality when moved to the setting where the scalar ring is merely assumed to be an integral domain. What is essential in Kouba's proof is commutativity of the scalar 
ring and absence of zero-divisors (any substantial weakening of these two assumptions seems to require to vary the argument substantially), and it is the purpose of the present note to try to lay bare the basic mechanism of Kouba's proof.

\section{Proof of Theorem 2}

The proof of the Theorem 2 will be based on the following simple lemma.

Lemma 3. Let $R$ be an integral domain. Let $\emptyset \neq S=\left\{s_{1}, \ldots, s_{m}\right\} \subseteq R$ be an arbitrary finite subset. Then there exist elements $\lambda_{1}^{(S)}, \ldots, \lambda_{m}^{(S)}$ of $R$ such that

$$
\begin{gathered}
\lambda_{1}^{(S)} \cdot\left(1, s_{1}, s_{1}^{2}, \ldots, s_{1}^{m-1}\right)+\cdots+\lambda_{m}^{(S)} \cdot\left(1, s_{m}, s_{m}^{2}, \ldots, s_{m}^{m-1}\right) \\
=\left(0,0,0, \ldots, 0, \prod_{1 \leqslant i<j \leqslant m}\left(s_{i}-s_{j}\right)\right) .
\end{gathered}
$$

(Note that for $m=1$ the claim is trivially true with $\lambda_{1}^{(S)}:=1$ and, as usual, taking the then empty product to be 1.)

Proof. Let $[m]:=\{1, \ldots, m\}$. Define $b$ to be the right-hand side of the claimed equation, taken as a column vector, and let $A=\left(a_{i j}\right)_{(i, j) \in[m]^{2}}$ be the Vandermonde matrix defined by $a_{i j}:=s_{j}^{i-1}$. Then the statement of the lemma is equivalent to the existence of a solution $\lambda^{(S)} \in R^{m}$ of the system of linear equations $A \lambda^{(S)}=b$. By the well-known formula for the determinant of a Vandermonde matrix (see [4], Ch. XIII, §4, example after Prop. 4.10), $\operatorname{det}(A)=\prod_{1 \leqslant i<j \leqslant m}\left(s_{i}-s_{j}\right)$.

Since $S$ is a set, all factors of this product are nonzero, and since $R$ has no zerodivisors, the determinant is therefore nonzero as well. Now let $\alpha_{i j}$ be the cofactors of $A$, i.e. $\alpha_{i j}:=(-1)^{i+j} \operatorname{det}\left(A^{(i j)}\right)$, where $A^{(i j)}$ is the $(m-1) \times(m-1)$ matrix obtained from $A$ by deleting the $i$-th row and the $j$-th column (see [2], Ch. IX, §3, before Lemma 1). By Cramer's rule (which is valid in any commutative ring, see Ch. IX, $\S 3$, Theorem 6 in [2] or Ch. XIII, $\S 4$, Theorem 4.4 in [4]), for every $j \in[m]$,

$$
\operatorname{det}(A) \cdot \lambda_{j}^{(S)}=\sum_{i=1}^{m} \alpha_{i j} b_{i} .
$$

Using $b_{m}=\operatorname{det}(A), b_{i}=0$ for every $1 \leqslant i<m$, and the commutativity of an integral domain, this reduces to

$$
\operatorname{det}(A) \cdot\left(\lambda_{j}^{(S)}-\alpha_{m j}\right)=0 .
$$

Hence, since $\operatorname{det}(A) \neq 0$ and $R$ has no zero-divisors, if follows that the cofactors $\lambda_{j}^{(S)}=\alpha_{m j} \in R$ provide explicit elements with the desired property.

Using this lemma, Kouba's argument may now be carried out without change in the setting of integral domains. 
Proof of Theorem 2. Let $R$ be an arbitrary integral domain and $f \in R\left[x_{1}, \ldots, x_{n}\right]$ be an arbitrary polynomial. Let $d_{1}, \ldots, d_{n} \geqslant 0$ be the exponents of a term $c \cdot x_{1}^{d_{1}} \cdots x_{n}^{d_{n}}$ with $c \neq 0$ which has maximum degree in $f$. For each $k \in[n]$, choose an arbitrary finite subset $S_{k} \subseteq R$ of size $d_{k}+1$ and apply Lemma 3 with $S=S_{k}$ and $m=|S|$ to obtain a family of elements $\left(\lambda_{s_{k}}^{\left(S_{k}\right)}\right)_{s_{k} \in S_{k}}$ of $R$ (where in order to avoid double indices the coefficients $\lambda$ are now being indexed by the elements of $S_{k}$ directly, not by an enumeration of each $S_{k}$ ) with the property that

$$
\begin{aligned}
& \sum_{s_{k} \in S_{k}} \lambda_{s_{k}}^{\left(S_{k}\right)} \cdot s_{k}^{\ell}=0 \quad \text { for every } \ell \in\left\{0, \ldots, d_{k}-1\right\}, \\
& \sum_{s_{k} \in S_{k}} \lambda_{s_{k}}^{\left(S_{k}\right)} \cdot s_{k}^{d_{k}}=\prod_{\left\{s, s^{\prime}\right\} \in\left(\begin{array}{c}
S_{k} \\
2
\end{array}\right)}\left(s-s^{\prime}\right)=: r_{k},
\end{aligned}
$$

where $\prod_{\left\{s, s^{\prime}\right\} \in\left(\begin{array}{c}S_{k} \\ 2\end{array}\right)}\left(s-s^{\prime}\right)=r_{k}$ is not a well-defined element of $R$ but only defined up to a sign $\left(\right.$ since $\left(s-s^{\prime}\right)=-\left(s^{\prime}-s\right)=(-1) \cdot\left(s^{\prime}-s\right)$ and $(-1) \cdot(-1)=-(-1)=1$, in every ring), depending on how the elements of $S_{k}$ are labelled. However, whatever specific labelling one chooses, $r_{k} \neq 0$ since $R$ does not have zero-divisors. Since the argument below does not make use of anything more specific about $r_{k}$ than its not being zero, it does not seem to be worthwhile to introduce a labelling of the elements of each $S_{k}$.

Now, using the coefficient families $\left(\lambda_{s_{k}}^{\left(S_{k}\right)}\right)_{s_{k} \in S_{k}}$, define, à la Kouba, the map

$$
\begin{aligned}
\Phi: \quad R\left[x_{1}, \ldots, x_{n}\right] \longrightarrow R & \quad \longmapsto \\
\qquad & \sum_{\left(s_{1}, \ldots, s_{n}\right) \in S_{1} \times \cdots \times S_{n}} \lambda_{s_{1}}^{\left(S_{1}\right)} \cdots \lambda_{s_{n}}^{\left(S_{n}\right)} \cdot g\left(s_{1}, \ldots, s_{n}\right) .
\end{aligned}
$$

Due to distributivity of - over + and commutativity of - in an integral domain, $\Phi$ is an $R$-linear form on the $R$-module $R\left[x_{1}, \ldots, x_{n}\right]$. In particular, for every polynomial $f$, the value $\Phi(f)$ can be evaluated termwise as

$$
\Phi(f)=\sum_{t \text { a term in } f} \Phi(t) .
$$

If $t=c \cdot x_{1}^{d_{1}^{\prime}} \cdots x_{n}^{d_{n}^{\prime}}$ is an arbitrary term in $R\left[x_{1}, \ldots, x_{n}\right]$, then

$$
\begin{aligned}
\Phi(t)=c \cdot \Phi\left(x_{1}^{d_{1}^{\prime}} \cdots x_{n}^{d_{n}^{\prime}}\right) & =c \cdot \sum_{\left(s_{1}, \ldots, s_{n}\right) \in S_{1} \times \cdots \times S_{n}} \lambda_{s_{1}}^{\left(S_{1}\right)} \cdots \lambda_{s_{n}}^{\left(S_{n}\right)} \cdot s_{1}^{d_{1}^{\prime}} \cdots s_{n}^{d_{n}^{\prime}} \\
& =c \cdot \sum_{s_{1} \in S_{1}} \cdots \sum_{s_{n} \in S_{n}} \lambda_{s_{1}}^{\left(S_{1}\right)} \cdots \lambda_{s_{n}}^{\left(S_{n}\right)} \cdot s_{1}^{d_{1}^{\prime}} \cdots s_{n}^{d_{n}^{\prime}} \\
& =c \cdot \prod_{k=1}^{n}\left(\sum_{s_{k} \in S_{k}} \lambda_{s_{k}}^{\left(S_{k}\right)} s_{k}^{d_{k}^{\prime}}\right),
\end{aligned}
$$

where in the last step again use has been made of the commutativity of an integral domain. By (6) and (2) it follows that for every term $t$, if there is at least one exponent $d_{i}^{\prime}$ with 
$d_{i}^{\prime}<d_{i}$, then $\Phi(t)=0$. Moreover, by the choice of the term $c \cdot x_{1}^{d_{1}} \cdots x_{n}^{d_{n}}$, every term $c^{\prime} \cdot x_{1}^{d_{1}^{\prime}} \cdots x_{n}^{d_{n}^{\prime}}$ of $f$ which is different from the term $c \cdot x_{1}^{d_{1}} \cdots x_{n}^{d_{n}}$ must, even if it has itself maximum degree in $f$, contain at least one exponent $d_{i}^{\prime}$ with $d_{i}^{\prime}<d_{i}$. Therefore

$$
\begin{aligned}
& \sum_{\left(s_{1}, \ldots, s_{n}\right) \in S_{1} \times \cdots \times S_{n}} \lambda_{s_{1}}^{\left(S_{1}\right)} \cdots \lambda_{s_{n}}^{\left(S_{n}\right)} \cdot f\left(s_{1}, \ldots, s_{n}\right) \stackrel{(4)}{=} \Phi(f) \stackrel{(5),(6),(2)}{=} c \cdot \Phi\left(x_{1}^{d_{1}} \cdots x_{n}^{d_{n}}\right)= \\
& \stackrel{(6),(3)}{=} c \cdot \prod_{k=1}^{n} \prod_{\left\{s, s^{\prime}\right\} \in\left(\begin{array}{c}
S_{k} \\
2
\end{array}\right)}\left(s-s^{\prime}\right)=c \cdot \prod_{k=1}^{n} r_{k} \neq 0,
\end{aligned}
$$

since $R$ has no zero-divisors. Obviously this implies that there exists at least one point $\left(s_{1}, \ldots, s_{n}\right) \in S_{1} \times \cdots \times S_{n}$ where $f$ does not vanish.

\section{Concluding question}

Is there any interesting use for the fact that even in the case of integral domains the coefficients of Kouba's map can be explicitly expressed in terms of cofactors of the matrices $\left(s_{j}^{i-1}\right) ?$

\section{Acknowledgements}

The author is very grateful to the department M9 of Technische Universität München for excellent working conditions, and to a referee for a remarkably careful report which pointed out quite a few inaccuracies.

\section{References}

[1] N. Alon, Combinatorial Nullstellensatz, Combin. Probab. Comput. 8 (1999), no. 1, $7-29$.

[2] G. D. Birkhoff and S. Mac Lane, Algebra, 3. ed., American Mathematical Society, 1987.

[3] O. Kouba, A duality based proof of the Combinatorial Nullstellensatz, Electron. J. Combin. 16 (2009), \#N9.

[4] S. Lang, Algebra, 3. ed., Graduate Texts in Mathematics, vol. 211, Springer, 2002.

[5] M. Michałek, A short proof of Combinatorial Nullstellensatz, arXiv:0904.4573v1 [math.CO] (2009).

[6] U. Schauz, Algebraically Solvable Problems: Describing Polynomials as Equivalent to Explicit Solutions, Electron. J. Combin. 15 (2008), \#R10. 